This item was submitted to Loughborough's Research Repository by the author.

Items in Figshare are protected by copyright, with all rights reserved, unless otherwise indicated.

\title{
Magnetic quantum oscillations in doped antiferromagnetic insulators
}

PLEASE CITE THE PUBLISHED VERSION

PUBLISHER

(C) American Physical Society

VERSION

VoR (Version of Record)

LICENCE

CC BY-NC-ND 4.0

REPOSITORY RECORD

Kabanov, V.V., and A.S. Alexandrov. 2019. "Magnetic Quantum Oscillations in Doped Antiferromagnetic Insulators". figshare. https://hdl.handle.net/2134/4249. 
This item was submitted to Loughborough's Institutional Repository (https://dspace.lboro.ac.uk/) by the author and is made available under the following Creative Commons Licence conditions.

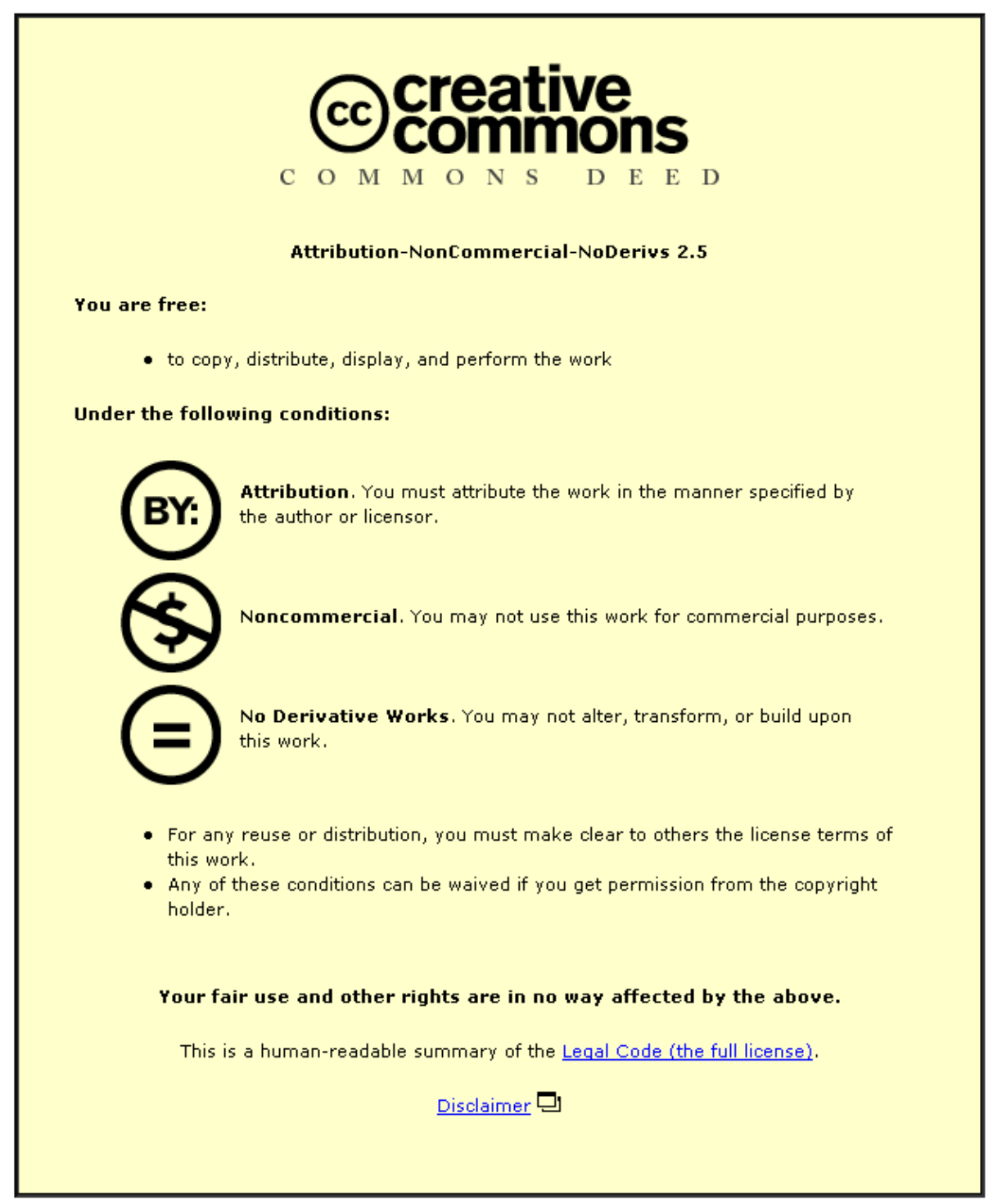

For the full text of this licence, please go to: http://creativecommons.org/licenses/by-nc-nd/2.5/ 


\title{
Magnetic quantum oscillations in doped antiferromagnetic insulators
}

\author{
V. V. Kabanov ${ }^{1,2}$ and A. S. Alexandrov ${ }^{2}$ \\ ${ }^{1}$ Jozef Stefan Institute, 1001 Ljubljana, Slovenia \\ ${ }^{2}$ Department of Physics, Loughborough University, Loughborough LE11 3TU, United Kingdom
}

(Received 1 February 2008; revised manuscript received 17 March 2008; published 10 April 2008)

\begin{abstract}
Energy spectrum of electrons (holes) doped into a two-dimensional antiferromagnetic insulator is quantized in an external magnetic field of arbitrary direction. A peculiar dependence of the de Haas-van Alphen or the Shubnikov-de Haas magneto-oscillation amplitudes on the azimuthal in-plane angle from the magnetization direction and on the polar angle from the out-of-plane direction is found, which can be used as a sensitive probe of the antiferromagnetic order in the doped Mott-Hubbard, spin-density wave, and conventional bandstructure insulators.
\end{abstract}

DOI: 10.1103/PhysRevB.77.132403

PACS number(s): 75.45. $+\mathrm{j}, 71.22 .+\mathrm{i}, 71.18 .+\mathrm{y}, 72.15 . \mathrm{Gd}$

Quantum oscillations of magnetization and resistivity with the magnetic field are of a great experimental and theoretical value providing reliable and detailed Fermi surfaces. ${ }^{1-3}$ Specifically, interest in the de Haas-van Alphen (dHvA) and the Shubnikov-de Haas $(\mathrm{SdH})$ effects in almost two-dimensional Fermi liquids has recently gone through a vigorous revival due to experimental discoveries of magnetooscillations in a few high-temperature cuprate superconductors. ${ }^{4-7}$ Their description in the framework of the standard theory for a metal ${ }^{1}$ has led to a small electronlike Fermi-surface area of a few percent of the first Brillouin zone and to a surprisingly low Fermi energy of about the room temperature, ${ }^{6,7}$ somewhat inconsistent with the firstprinciples (local density approximation) band structures and angle-resolved photoemission spectroscopy spectra of cuprates. ${ }^{8}$ The oscillations have been observed in the superconducting (vortex) state well below the upper critical field raising a doubt concerning their normal state origin. ${ }^{9}$ While a better understanding of the $\mathrm{dHvA} / \mathrm{SdH}$ effects in doped antiferromagnetic insulators is generally important, it becomes particularly vital for building an adequate theory of hightemperature superconductivity since parent cuprates are antiferromagnets.

Here, using a tight-binding Hamiltonian, we quantize the energy spectrum of electrons or holes moving on the antiferromagnetic background in a two-dimensional lattice. We find a peculiar dependence of the magneto-oscillation amplitudes on the magnetic field direction, which could serve as a sensitive probe of the antiferromagnetic order in doped insulators.

The mean-field tight-binding Hamiltonian of carriers doped into the bipartite antiferromagnetic square lattice in the external magnetic field $\mathbf{B}$ is written as

$$
\begin{aligned}
H= & \sum_{i i^{\prime}} \delta_{i i^{\prime}}\left(\Delta \hat{a}_{i}^{\dagger} \sigma_{z} \hat{a}_{i}+\mu_{B} \mathbf{B} \hat{a}_{i}^{\dagger} \boldsymbol{\sigma} \hat{a}_{i}\right)+t_{i i^{\prime}} \hat{a}_{i^{\prime}}^{\dagger} \hat{a}_{i} \\
& -\sum_{j j^{\prime}} \delta_{j j^{\prime}}\left(\Delta \hat{b}_{j}^{\dagger} \sigma_{z} \hat{b}_{j}-\mu_{B} \mathbf{B} \hat{b}_{j}^{\dagger} \boldsymbol{\sigma} \hat{b}_{j}\right)+t_{j j^{\prime}} \hat{b}_{j^{\prime}}^{\dagger} \hat{b}_{j}+\sum_{i j} t_{i j} \hat{b}_{j}^{\dagger} \hat{a}_{i} \\
& + \text { H.c. },
\end{aligned}
$$

where $\hat{a}_{i}^{\dagger}=\left(a_{i \uparrow}^{\dagger}, a_{i \downarrow}^{\dagger}\right)$ and $\hat{b}_{j}^{\dagger}=\left(b_{j \uparrow}^{\dagger}, b_{j \downarrow}^{\dagger}\right)$ create the carrier on sites $i$ and $j$ of sublattices $A$ and $B$, respectively, with the spin $s=\uparrow, \downarrow, \Delta$ is the carrier spin-lattice spin exchange energy (the antiferromagnetic gap), $\delta_{i i^{\prime}}$ is the Kroneker symbol, $t_{i i^{\prime}}$, $t_{j j^{\prime}}$, and $t_{i j}$ are the hopping integrals, and $\boldsymbol{\sigma} \equiv\left\{\sigma_{x}, \sigma_{y}, \sigma_{z}\right\}$ are the Pauli matrices.

Fourier transforming the operators from the Wannier (site) to the Bloch (momentum, k) representation and assuming translational invariance, the carrier energy spectrum $E(\mathbf{k})$ is found by diagonalizing a $4 \times 4$ matrix,

$$
\left(\begin{array}{cc}
t_{\mathbf{k}}^{\prime}-\Delta \sigma_{0}-\mu_{B} B_{\|} \sigma_{z} & \mu_{B} B_{\perp} \sigma_{0}+t_{\mathbf{k}} \sigma_{x} \\
\mu_{B} B_{\perp} \sigma_{0}+t_{\mathbf{k}} \sigma_{x} & t_{\mathbf{k}}^{\prime}+\Delta \sigma_{0}+\mu_{B} B_{\|} \sigma_{z}
\end{array}\right)
$$

where $t_{\mathbf{k}}^{\prime}=\sum_{j j^{\prime}} t_{j j^{\prime}} \exp \left(i\left[\mathbf{k} \cdot\left(\mathbf{j}^{\prime}-\mathbf{j}\right)\right]\right)$ is the hopping energy within one sublattice and $t_{\mathbf{k}}=\sum_{i j} t_{i j} \exp (i[\mathbf{k} \cdot(\mathbf{i}-\mathbf{j})])$ is the intersublattice hopping energy. This matrix corresponds to the choice of the four-dimensional vector in the spin and sublattice space at fixed $\mathbf{k}$. Here, $B_{\perp}$ and $B_{\|}$are transverse and longitudinal components of the magnetic field with respect to the lattice magnetization $\mathbf{n}$ (Fig. 1), $\sigma_{0}$ is the identity matrix, and $\mu_{B}$ is the Bohr magneton. There are two electron and two hole bands dispersed as

$$
E(\mathbf{k})=t_{\mathbf{k}}^{\prime} \pm\left[\Delta^{2}+t_{\mathbf{k}}^{2}\right]^{1 / 2} \pm \mu_{B}\left[B_{\|}^{2}+\frac{t_{\mathbf{k}}^{2}}{\Delta^{2}+t_{\mathbf{k}}^{2}} B_{\perp}^{2}\right]^{1 / 2}
$$

in the lowest order with respect to the field $\mu_{B} B \ll \Delta$. They are split by the external magnetic field into two subbands each with anisotropic $g$ factor, $g=2\left[\cos ^{2}(\phi)+\sin ^{2}(\phi) t_{\mathbf{k}}^{2} /\left(\Delta^{2}\right.\right.$ $\left.+t_{\mathbf{k}}^{2}\right)$, depending on the angle $\phi$ between the field and the magnetization (Fig. 1).

The anisotropic $g$ factor differs significantly from the free electron $g_{e}=2$ near the extremum points of the valence

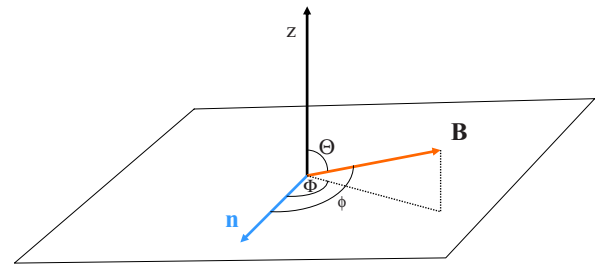

FIG. 1. (Color online) The azimuthal in-plane angle $\Phi$ from the magnetization direction $\mathbf{n}$ and the polar angle $\Theta$ of the magnetic field $\mathbf{B}$ from the out-of-plane direction. 

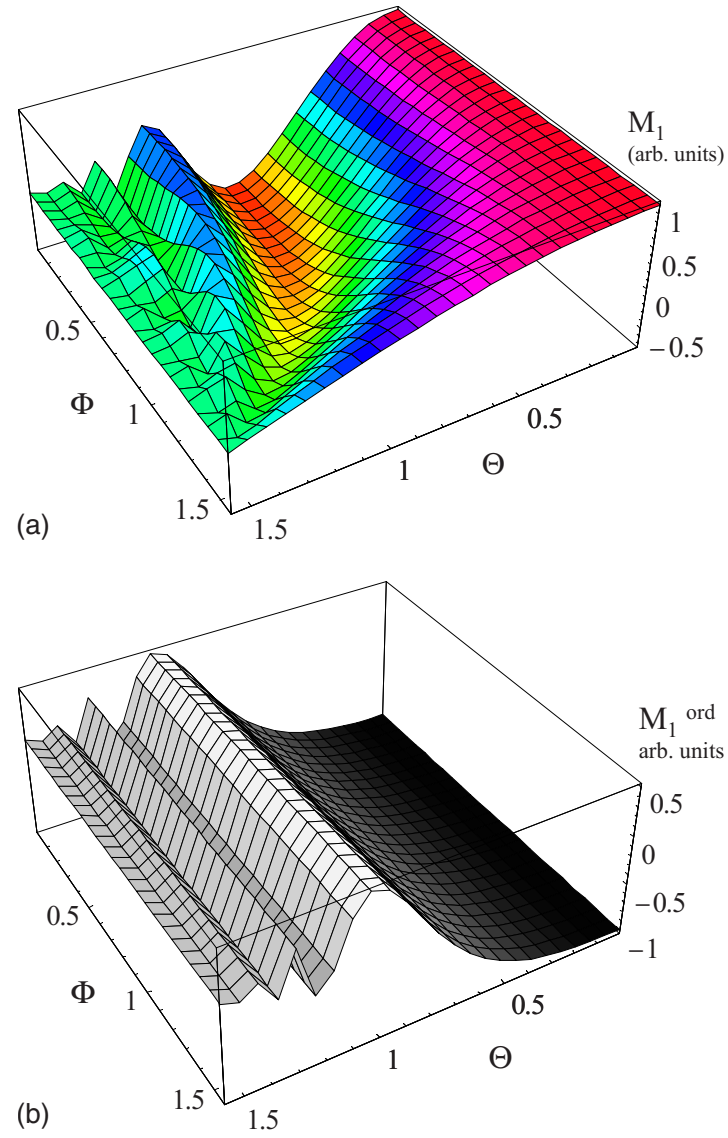

FIG. 2. (Color online) dHvA first-harmonic amplitude as a function of the azimuthal in-plane angle from the magnetization direction $\Phi$ and the polar angle from the out-of-plane direction $\Theta$ in a layered antiferromagnet (upper panel) compared to the firstharmonic amplitude in a nonmagnetic layered metal (lower panel) at $T=\Gamma=0$ and $\left(m_{x} m_{y}\right)^{1 / 2}=m_{e}$.

and/or conductance bands, where $t_{\mathbf{k}}^{2} \ll \Delta^{2}$. According to the general principles of quantum mechanics, deviations of the $g$ factor from its classical value are related to spin-orbit interaction. The spin-orbit interaction is not included explicitly to the Hamiltonian [Eqs. (1) and (2)]. Basically, the difference originates from the spin-orbit interaction pinning the lattice magnetization along a crystal lattice direction and implicitly present in the Hamiltonian. At a relatively low doping with the Fermi energy $E_{F}$ near the top (bottom) of the valence (conduction) band, one can expand Eq. (3) in powers of $t / \Delta$,

$$
E(\mathbf{k}) \approx \frac{\hbar^{2} k_{x}^{2}}{2 m_{x}}+\frac{\hbar^{2} k_{y}^{2}}{2 m_{y}} \pm \mu_{B}\left[B_{\|}^{2}+\gamma^{2}(\mathbf{k}) B_{\perp}^{2}\right]^{1 / 2} .
$$

Here, $m_{x}^{-1}=4 a^{2}\left(2 t^{2} / \Delta-t^{\prime}\right) / \hbar^{2}$ and $m_{y}^{-1}=4 a^{2} t^{\prime} / \hbar^{2}$ are the components of the effective mass tensor, $a$ is the lattice constant, $t$ and $t^{\prime}>0$ are nearest and nearest next neighbor hopping integrals, respectively, and the coefficient $\gamma(\mathbf{k})$ is small as $\gamma(\mathbf{k})=2 \sqrt{2} t k_{x} / \Delta \sim\left(E_{F} / \Delta\right)^{1 / 2} \ll 1$. Here, $k_{x}$ and $k_{y}$ are deviations of the wave vector from the extremum point perpendicular and parallel to the antiferromagnetic Brillouin zone boundary, respectively, and the energy of the extremum point is taken as zero.

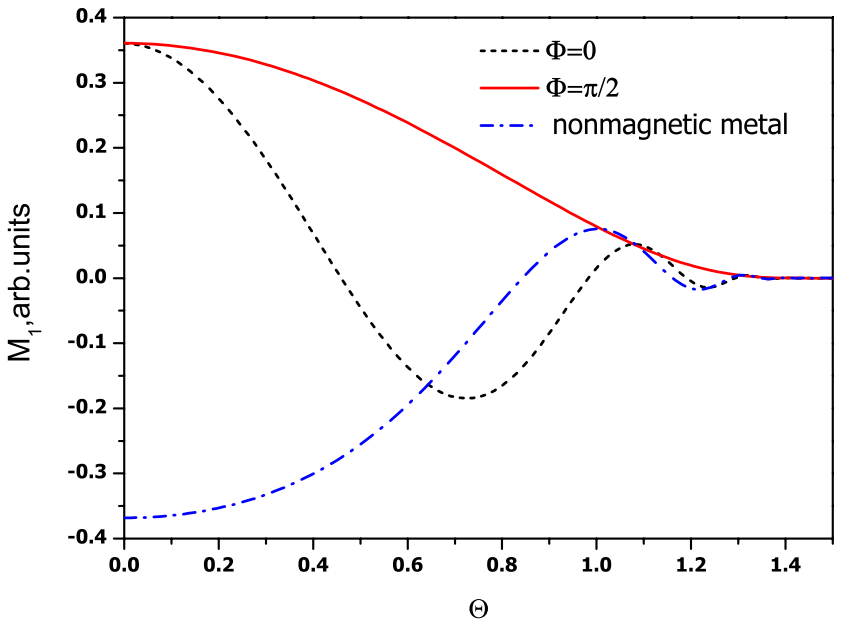

FIG. 3. (Color online) dHvA first-harmonic amplitude as a function of the polar angle $\Theta$ for two different azimuthal in-plane angles $\Phi$ in a layered antiferromagnet compared to the $\Phi$-independent first-harmonic amplitude in a nonmagnetic layered metal at $T=0$, $2 \pi \Gamma=\hbar \omega, \gamma k_{F}=0.1$, and $\left(m_{x} m_{y}\right)^{1 / 2}=m_{e}$.

The anisotropic $g$ factor in doped antiferromagnetic insulators was originally derived in a weak-coupling nesting model. ${ }^{10}$ Actually, the effective mass approximation [Eq. (4)] can also be derived phenomenologically by using the symmetry arguments. ${ }^{11}$ The nonunitary group of the antiferromagnetic lattice is $G=\left\{D_{4}, R \mathbf{T}\right\}$, where $D_{4}$ describes all rotations which remain the system invariant. Translations $\mathbf{T}$ by a lattice period transform from one sublattice to another, changing the sign of the magnetization. Hence, these translations are multiplied by the time inversion operator $R$. Following Brazovskii and Lukyanchuk, ${ }^{11}$ one can construct the Hamiltonian of the required symmetry as

$$
\begin{aligned}
H= & \left(\frac{\hbar^{2} k_{x}^{2}}{2 m_{x}}+\frac{\hbar^{2} k_{y}^{2}}{2 m_{y}}\right) \hat{a}^{\dagger} \sigma_{0} \hat{a} \\
& +\mu_{B}\left[(\mathbf{B} \cdot \mathbf{n}) \hat{a}^{\dagger}(\mathbf{n} \cdot \boldsymbol{\sigma}) \hat{a}+\gamma(\mathbf{k})(\mathbf{B} \times \mathbf{n}) \hat{a}^{\dagger}(\mathbf{n} \times \boldsymbol{\sigma}) \hat{a}\right],
\end{aligned}
$$

with the electron (hole) energy spectrum [Eq. (4)]. Here, $\mathbf{n}$ is the magnetization unit vector and $\hat{a}^{\dagger}=\left(a_{\uparrow}^{\dagger} a_{\dagger}^{\dagger}\right), \hat{a}$ are creation and annihilation operators, respectively, for the spinor describing the hole (electron) band. The coefficient $\gamma(\mathbf{k})$ is an odd function of $k_{x}$, which is zero at the antiferromagnetic Brillouin zone boundary, so that $\gamma(\mathbf{k})=\gamma k_{x}$, where $\gamma$ does not depend on $\mathbf{k}$. The coupling to the magnetic field in this Hamiltonian is obtained noticing that the transformation $\mathbf{k}$ $\rightarrow \mathbf{k}+\mathbf{Q}$, with $\mathbf{Q}=\pi \mathbf{a} / a^{2}$ is equivalent to the rotation in the spinor space described by the matrix $\mathbf{n} \cdot \boldsymbol{\sigma}$ (Ref. 11) (here, $\mathbf{a}=\{a, a\}$ ). Direct comparison of the eigenvalues of the Hamiltonian [Eq. (5)] with the spectrum [Eq. (3)] yields $\gamma$ $=2 \sqrt{2} t / \Delta$. Importantly, the symmetry arguments are applied beyond the mean-field approximation [Eq. (1)] so that spin fluctuations just renormalize the effective mass tensor and other coefficients in Eq. (4).

The orbital quantization of the spectrum [Eq. (3)] is readily obtained via the Peierls substitution, ${ }^{12} \mathbf{k} \Rightarrow-i \nabla+e \mathbf{A}$, 


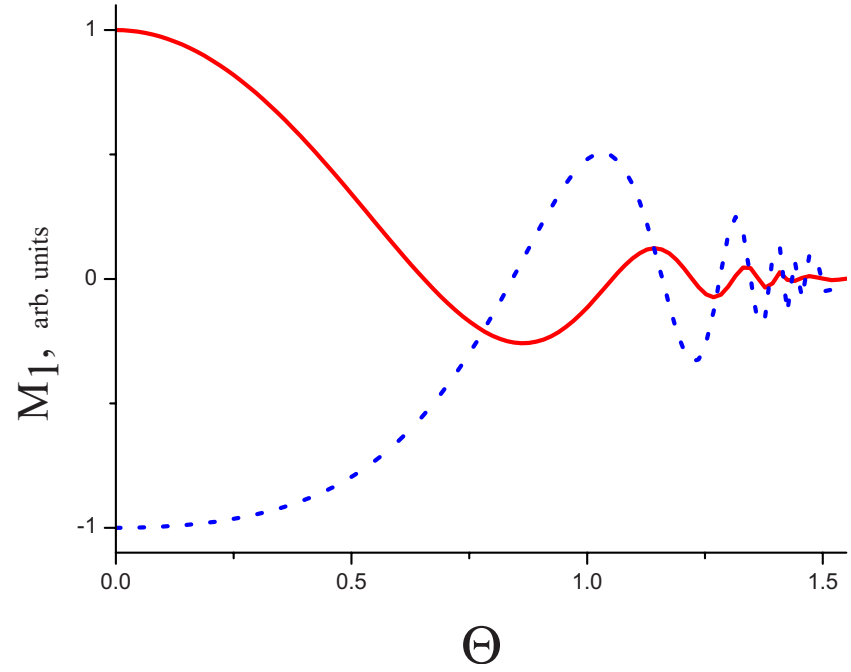

FIG. 4. (Color online) dHvA first-harmonic amplitude as a function of the polar angle $\Theta$ in a disordered antiferromagnet (solid line) compared to the first-harmonic amplitude in a nonmagnetic layered metal (dotted line) at $T=\Gamma=0$ and $\left(m_{x} m_{y}\right)^{1 / 2}=m_{e}$.

with the vector potential $\mathbf{A}(\mathbf{r})$ in Eq. (2). In the lowest order with respect to $E_{F} / \Delta$, we can use the effective mass approximation [Eq. (4)], which yields the conventional FockLandau level ${ }^{13,14}$ split by the longitudinal field as

$$
E_{n}=\hbar \omega|\cos (\Theta)|(n+1 / 2) \pm \mu_{B}\left|B_{\|}\right|,
$$

where $\omega=e B /\left(m_{x} m_{y}\right)^{1 / 2}$ is the cyclotron frequency, $n$ $=0,1,2 \ldots$, and $\Theta$ is the polar angle between the magnetic field and the out-of-plane direction (Fig. 1).

Now, the oscillating part of the magnetization per unit volume $\tilde{M}$ is calculated following the standard route by applying the Poisson summation: ${ }^{1}$

$$
\tilde{M}=\sum_{r=1}^{\infty} M_{r} \sin \frac{2 \pi r F}{B} .
$$

Here,

$$
M_{r}=A_{r}(\Theta) \cos \left[\frac{\pi r\left(m_{x} m_{y}\right)^{1 / 2} \tan (\Theta) \cos (\Phi)}{m_{e}}\right]
$$

is the amplitude of $r$ harmonic, with

$$
\begin{aligned}
A_{r}(\Theta)= & (-1)^{r+1} \frac{e E_{F} \cos (\Theta)}{2 \pi^{2} \hbar d r} \\
& \times R_{T}\left(\frac{2 \pi^{2} r k_{B} T}{\hbar \omega \cos (\Theta)}\right) R_{D}\left(\frac{2 \pi r \Gamma}{\hbar \omega \cos (\Theta)}\right),
\end{aligned}
$$

$F=\left(m_{x} m_{y}\right)^{1 / 2} E_{F} / e \hbar \cos (\Theta)$ is the fundamental frequency of oscillations, $R_{T}(z)=z / \sinh (z)$ and $R_{D}(z)=\exp (-z)$ are conventional temperature and the Dingle reduction factors, $\Gamma / \hbar$ is the scattering rate, $m_{e}$ is the free electron mass, $d$ is the interplane distance, and $\Phi$ is the azimuthal in-plane angle from the magnetization direction (Fig. 1). Both angles $\Theta$ and $\Phi$ in Eq. (8) are changing in the interval $0 \leqslant \Theta, \Phi \leqslant \pi / 2$. Three-dimensional corrections to the energy spectrum can be accounted for by the additional Yamaji factor, ${ }^{15} R_{Y}$
$=J_{0}\left[4 \pi r \tilde{t}_{\perp} / \hbar \omega \cos (\Theta)\right]$ in Eq. (9), where $J_{0}(x)$ is the zeroorder Bessel function, $\tilde{t}_{\perp}=t_{\perp} J_{0}\left[k_{F} d \tan (\Theta)\right], t_{\perp}$ is the out-ofplane hopping integral, and $\hbar k_{F}$ is the Fermi momentum.

As follows from Eq. (8), the essential anisotropy of the $g$ factor causes a strong dependence of the oscillation amplitude on the azimuthal in-plane angle of the field from the magnetization direction [Fig. 2(a)], which is absent in the ordinary nonmagnetic layered metals [Fig. 2(b)], where the magnetization amplitudes are found as

$$
M_{r}^{\text {ord }}=A_{r}(\Theta) \cos \left[\frac{\pi r\left(m_{x} m_{y}\right)^{1 / 2}}{m_{e} \cos (\Theta)}\right]
$$

The dependence on $\Phi$ and $\Theta$ [Eq. (8)] is extremely pronounced at low temperatures (compare upper panel and lower panel of Fig. 2), as also shown in Fig. 3 for some fixed azimuthal angles.

One can readily generalize our results to any shape of the Fermi surface and calculate corrections to amplitudes and fundamental frequencies of higher order in $E_{F} / \Delta$ and in the magnetic field by applying the Lifshits-Kosevich quasiclassical approximation. ${ }^{16}$ Within the approximation, dHvA frequencies $F_{ \pm}$are determined by the extremal cross-section areas, with $S_{ \pm}^{\text {ext }}$ of the two spin-split electron (or hole) Fermi surfaces, $F_{ \pm}=\hbar S_{ \pm}^{\text {ext }} / 2 \pi e$. Following Ref. 17, one can expand the extremal cross-section area in powers of the magnetic field, so that $F_{ \pm}=F \pm \alpha B+\beta B^{2} \pm \epsilon B^{3}$. Here, the second term describes the Zeeman splitting of the bands with the anisotropic $g$ factor. It does not shift the frequency but affects the amplitude. The third term describes a small shift of the fundamental frequency $F$ depending on the magnetic field. The last term describes a small field-dependent correction to the $g$ factor. For example, when the field is perpendicular to the magnetization, $B_{\|}=0$, and the effective mass approximation is applied near the $X$ point, $(\pi / 2 a, \pi / 2 a)$, of the antiferromagnetic Brillouin zone, one finds $F=\hbar k_{F}^{2} / 2 e, \quad \alpha$ $=\left(m_{x} m_{y}\right)^{1 / 2} \gamma k_{F} / \pi m_{e}, \quad \beta=m_{x}^{3 / 2} m_{y}^{1 / 2} \gamma^{2} e / 8 m_{e}^{2} \hbar, \quad$ and $\epsilon$ $=m_{x}^{9 / 4} m_{y}^{3 / 4} \gamma^{3} e^{2} / 12 \pi m_{e}^{3} \hbar^{2} k_{F}$, with $\hbar k_{F}=\left[2\left(m_{x} m_{y}\right)^{1 / 2} E_{F}\right]^{1 / 2}$. For an arbitrary field direction, one obtains, using Eq. (4) with $\gamma(\mathbf{k})=\gamma k_{x} \ll 1$,

$$
\frac{M_{r}}{A_{r}(\Theta)}=\cos \left[\frac{2 r\left\{m_{x} m_{y}\left[\cos ^{2}(\phi)+\gamma^{2} k_{F}^{2} \sin ^{2}(\phi)\right]\right\}^{1 / 2} E[\kappa(\phi)]}{m_{e} \cos (\Theta)}\right] .
$$

Here, $\phi$ is the angle between the magnetic field and the magnetization (Fig. 1), $E(\kappa)$ is the elliptic integral of the second kind, and

$$
\kappa(\phi)=\left[\frac{\gamma^{2} k_{F}^{2} \sin ^{2}(\phi)}{\cos ^{2}(\phi)+\gamma^{2} k_{F}^{2} \sin ^{2}(\phi)}\right]^{1 / 2} .
$$

Taking $\gamma=0$ in Eq. (11), one obtains Eq. (8), since $\cos ^{2}(\phi)$ $=\sin ^{2}(\Theta) \cos ^{2}(\Phi)$ and $E[0]=\pi / 2, E[1]=1$. The finite transverse spin susceptibility, $\propto \gamma k_{F}=\left(2 E_{F} / \Delta\right)^{1 / 2}$, only slightly blurs the strong $\Phi$ dependence of the amplitudes (Fig. 3) if 
$E_{F} / \Delta \ll 1$. For example, when the field is rotated in the plane perpendicular to the magnetization axis $\mathbf{n}$, we have

$$
M_{r}=A_{r}(\Theta) \cos \left[\frac{2 r\left(2 m_{x} m_{y} E_{F} / \Delta\right)^{1 / 2}}{m_{e} \cos (\Theta)}\right]
$$

with a small transverse $g$ factor. On the other hand, if the magnetic field is rotating in the $(z, \mathbf{n})$ plane, the angular dependence is quite different,

$$
M_{r}=A_{r}(\Theta) \cos \left[\frac{\pi r\left(m_{x} m_{y}\right)^{1 / 2} \tan (\Theta)}{m_{e}}\right],
$$

as in Eq. (8) with $\Phi=0$.

Real antiferromagnetic solids, such as cuprates, could be disordered or twinned, so that the magnetization direction $\mathbf{n}$ within the plane is random. Nevertheless, the dependence of dHvA amplitudes on the polar angle $\Theta$ remains rather unconventional. Indeed, averaging Eq. (8) over all directions of $\Phi$ from 0 to $\pi / 2$ yields

$$
\left\langle M_{r}\right\rangle=A_{r}(\Theta) J_{0}\left[\frac{\pi r\left(m_{x} m_{y}\right)^{1 / 2} \tan (\Theta)}{m_{e}}\right],
$$

which is distinguishably different from the amplitudes in a nonmagnetic metal [Eq. (10)] (Fig. 4). There are known relations between oscillations in transport and thermodynamic quantities $^{1}$ at least in nonmagnetic substances. Relying on them, we expect the similar nontrivial angle dependences also in the $\mathrm{SdH}$ magneto-oscillations.

In summary, we have derived the energy spectrum of electrons (holes) doped into a two-dimensional antiferromagnetic insulator in terms of all-neighbor hopping integrals of nonmagnetic lattice [Eq. (3)] and quantized it in the external magnetic field of arbitrary direction. The peculiar dependence of $\mathrm{dHvA} / \mathrm{SdH}$ magneto-oscillation amplitudes on the azimuthal in-plane angle from the magnetization direction and on the polar angle from the out-of-plane direction is found, which could be instrumental as a sensitive probe of the antiferromagnetic order in the doped Mott-Hubbard, spin-density wave, and conventional band-structure insulators.

We greatly appreciate valuable discussions with Revaz Ramazashvili and Iorwerth Thomas and support of this work by EPSRC (UK) (Grant No. EP/D035589).
${ }^{1}$ D. Schoenberg, Magnetic Oscillations in Metals (Cambridge University Press, Cambridge, 1984).

${ }^{2}$ J. Singleton, Rep. Prog. Phys. 63, 1111 (2000).

${ }^{3}$ M. V. Kartsovnik, Chem. Rev. (Washington, D.C.) 104, 5737 (2004), and references therein.

${ }^{4}$ N. Doiron-Leyraud, C. Proust, D. LeBoef, J. Levalois, J.-B. Bonnemaison, R. Liang, D. A. Bonn, W. N. Hardy, and L. Taillefer, Nature (London) 447, 565 (2007).

${ }^{5}$ A. F. Bangura, J. D. Fletcher, A. Carrington, J. Levallois, M. Nardone, B. Vignolle, P. J. Heard, N. Doiron-Leyraud, D. LeBoeuf, L. Taillefer, S. Adachi, C. Proust, and N. E. Hussey, Phys. Rev. Lett. 100, 047004 (2008).

${ }^{6}$ E. A. Yelland, J. Singleton, C. H. Mielke, N. Harrison, F. F. Balakirev, B. Dabrowski, and J. R. Cooper, Phys. Rev. Lett. 100, 047003 (2008).

${ }^{7}$ C. Jaudet, D. Vignolles, A. Audouard, J. Levallois, D. LeBoeuf, N. Doiron-Leyraud, B. Vignolle, M. Nardone, A. Zitouni, R.
Liang, D. A. Bonn, W. N. Hardy, L. Taillefer, C. Proust, arXiv:0711.3559 (unpublished).

${ }^{8}$ A. Damascelli, Z. Hussain, and Zhi-Xun Shen, Rev. Mod. Phys. 75, 473 (2003).

${ }^{9}$ A. S. Alexandrov, arXiv:0711.0093 (unpublished).

${ }^{10}$ S. A. Brazovskii, I. A. Lukyanchuk, and R. R. Ramazashvili, Jr., Pis'ma Zh. Eksp. Teor. Fiz. 49, 557 (1989) [JETP Lett. 49, 644 (1989)].

${ }^{11}$ S. A. Brazovskii and I. A. Lukyanchuk, Zh. Eksp. Teor. Fiz. 96, 2088 (1989) [Sov. Phys. JETP 69, 1180 (1989)].

${ }^{12}$ R. E. Peierls, Z. Phys. 80, 763 (1933).

${ }^{13}$ V. Fock, Z. Phys. 47, 446 (1928).

${ }^{14}$ L. Landau, Z. Phys. 64, 629 (1930).

${ }^{15}$ K. Yamaji, J. Phys. Soc. Jpn. 58, 1520 (1989).

${ }^{16}$ J. M. Lifshits and A. M. Kosevich, Zh. Eksp. Teor. Fiz. 29, 730 (1955) [Sov. Phys. JETP 2, 636 (1956)].

${ }^{17}$ V. P. Mineev and K. V. Samokhin, Phys. Rev. B 72, 212504 (2005). 